\title{
Aspectos que influyen en la consolidación de empresas: evidencias obtenidas en 14 países
}

\section{Factors influencing on entrepreneurial consolidation: evidence from 14 countries}

\author{
Agustín Álvarez-Herranz $\quad$ Pilar Valencia-De-Lara² $\quad$ María Pilar Martínez-Ruiz ${ }^{3}$ \\ Recibido 28 de octubre de 2010, aceptado 13 de abril de 2011 \\ Received: October 28, $2010 \quad$ Accepted: April 13, 2011
}

\begin{abstract}
RESUMEN
Este trabajo analiza el efecto que las motivaciones del emprendedor -concretamente, ciertas características del emprendedor y de su entorno- ejercen en la consolidación empresarial. Con este propósito se ha analizado una base de datos de series temporales y de corte transversal relativos a una muestra de emprendedores obtenida en catorce países. Los resultados logrados evidencian la existencia de una relación positiva entre la consolidación empresarial y el hecho de que el emprendedor: (1) considere que crear una empresa es una buena opción profesional; (2) perciba mayor riesgo y (3) ponga en marcha su negocio basándose en la detección de oportunidades. Estos hallazgos han permitido inferir una serie de implicaciones relevantes tanto para la gestión empresarial como para las políticas de desarrollo local/regional.
\end{abstract}

Palabras clave: Emprendedor, consolidación empresarial, enfoque multipaís, motivaciones, proyecto GEM.

\begin{abstract}
This work analyzes the effect that entrepreneurial motivations -in particular, certain entrepreneur and environment characteristics -have on business consolidation. Bearing this goal in mind, a panel and longitudinal database referring to a sample of entrepreneurs obtained from fourteen countries has been analyzed. The results evidence the existence of a positive relationship between business consolidation and the fact that the entrepreneur: (1) thinks that starting up a business is a good professional option; (2) perceives higher risk and (3) sets up his/her business detecting opportunities. These findings enable the development of relevant implications for both the business management and local/regional authorities.
\end{abstract}

Keywords: Entrepreneur, business consolidation, multicountry perspective, motivations, GEM project.

\section{INTRODUCCIÓN}

La literatura relevante en el ámbito de la creación de empresas ha mostrado cómo las motivaciones que fomentan la creación y puesta en marcha de un negocio se encuentran estrechamente relacionadas con ciertos rasgos del emprendedor, así como con algunos factores del entorno. Sin embargo, no resultan abundantes los trabajos que hayan abordado la influencia de los aspectos anteriores en la consolidación empresarial.

Por este motivo, esta investigación pretende determinar si las motivaciones del emprendedor -relacionadas con ciertos rasgos del emprendedor así como con determinadas oportunidades de su

1 Área de Econometría. Facultad de Ciencias Económicas y Empresariales. Universidad de Castilla-La Mancha. Plaza de la Universidad, s/n. 02071 Albacete (España). Email: Agustin.Alvarez@uclm.es

2 Área de Organización de Empresas. Facultad de Ciencias Sociales de Cuenca. Universidad de Castilla-La Mancha. Avenida de los Alfares, 44. 16071 Cuenca (España). Email: Pilar.Valencia@uclm.es

3 Área de Comercialización e Investigación de Mercados. Facultad de Ciencias Sociales de Cuenca. Universidad de Castilla-La Mancha. Avenida de los Alfares, 44.16071 Cuenca (España). Email: MariaPilar.Martinez@uclm.es 
entorno- influyen en la consolidación empresarial, entendiendo como empresarios consolidados la definición dada por el proyecto GEM de "propietarios gerentes de actividades empresariales que llevan establecidas más de 42 meses". Y todo ello, en un contexto específico que confiere mayor atractivo al presente trabajo: los países que componen el proyecto Global Entrepreneurship Monitor (en adelante GEM). Este entorno ha sido seleccionado para desarrollar el estudio en virtud tanto de la relevancia que el proyecto GEM ha adquirido en la actualidad en el ámbito de la creación de empresas como de la posibilidad de obtener gran cantidad de datos actualizados sobre diversas variables del empresario emprendedor y de su entorno.

En la sección siguiente se describirá el marco teórico de la presente investigación, lo que permitirá posteriormente presentar las principales conclusiones obtenidas en el estudio empírico realizado.

\section{MARCO CONCEPTUAL}

Entre las motivaciones del emprendedor que favorecen la creación y puesta en marcha de un negocio la revisión de la literatura relevante ha destacado la imagen social del empresario, que constituye un factor clave para que surjan nuevos empresarios en una sociedad [1-6]. Imagen que normalmente se encuentra ligada a la combinación de los valores culturales y a la ideología imperante en una sociedad [7], y que es susceptible de fomentar el desarrollo de proyectos empresariales en tanto en cuanto pone de manifiesto aspectos que resultan deseables en una sociedad (e.g. [8-9]).

Es importante considerar además la legitimación social de esta imagen para comprender el mayor o menor impacto que la misma pueda ejercer sobre la motivación de crear un proyecto empresarial. En efecto, la percepción del propio individuo de que las normas y valores sociales dominantes consideran legítima la actividad empresarial, puede suponer un estímulo para la creación de empresas [9-10]. Por el contrario, las percepciones y las actitudes negativas de una sociedad respecto a la imagen social del empresario pueden inhibir la creación de empresas, especialmente en aquellas situaciones en las que existe un menor desarrollo del entorno empresarial (e.g. [11]).
Por otro lado, la propensión a asumir riesgos -tanto de tipo financiero, como en lo relativo a la reputación social, etc.- ha sido tradicionalmente considerada como una de las características del empresario que pueden influir en mayor medida en la creación y puesta en marcha de una empresa (e.g. [12-16]). Al respecto, los trabajos previos realizados en esta línea de investigación no son concluyentes, mostrando cómo mientras en algunos casos los empresarios fundadores poseen una orientación moderada al riesgo (e.g. [17-19]), en otros poseen una mayor propensión al riesgo frente a otros individuos [13].

Ahora bien, en relación con estos últimos empresarios no está claro si esta propensión más elevada puede deberse a que perciben el riesgo de manera diferente $[12,14]$, por lo que la diferencia entre empresarios y no empresarios podría radicar en la forma de clasificar las situaciones a las que se enfrentan, percibiendo de este modo más fortalezas y oportunidades que amenazas y debilidades [14].

Finalmente, el reconocimiento de oportunidades de negocio por parte del emprendedor puede proporcionarle la capacidad de detectar oportunidades en su entorno que probablemente hayan pasado desapercibidas previamente a otros individuos [20-23, 16]. En efecto, frente a algunos investigadores que consideran que la innovación sistemática es la función concreta del espíritu empresarial [24-28], para otros es precisamente el descubrimiento y la explotación de oportunidades donde reside la esencia de la actividad empresarial [21-23, 29]. No en vano, el reconocimiento de estas oportunidades puede constituir la posibilidad de crear el vínculo entre la necesidad de mercado insatisfecha y la solución para satisfacer dicha necesidad (e.g. [30]).

$\mathrm{Al}$ respecto, cabe señalar que aunque las investigaciones de mercados pueden ser útiles a la hora de reconocer ciertas oportunidades, la intuición basada en la experiencia puede tener una mayor importancia en el reconocimiento de los problemas del mercado como fuente de oportunidades [31]. De ahí que el conocimiento de los problemas comerciales y la sensibilidad del individuo para reconocer oportunidades de mercado han sido frecuentemente asociados a la creación de empresas $[27,31]$. 
No obstante, detectar problemas en el mercado en ocasiones puede no resultar una cuestión sencilla [32], bien porque las necesidades insatisfechas de los demandantes se consideren normales debido a la costumbre, o bien porque los oferentes, debido a la falta de capacidad de observación y de análisis crítico, sean incapaces de ofrecer una solución a los consumidores [27].

Teniendo en cuenta las ideas previas, el Cuadro 1 sintetiza los hallazgos principales que se han obtenido en el marco del proyecto GEM en relación con las motivaciones del emprendedor anteriormente comentadas que son susceptibles de fomentar la creación y puesta en marcha de un negocio.

Cuadro 1. Principales resultados obtenidos en el marco del proyecto GEM.

\begin{tabular}{|l|l|}
\hline Aspecto & Hallazgos obtenidos \\
\hline $\begin{array}{l}\text { La imagen social } \\
\text { del empresario }\end{array}$ & $\begin{array}{l}\text { Entre los principales obstáculos detectados } \\
\text { para la creación de empresas, destacan los } \\
\text { valores culturales, las normas sociales y } \\
\text { las políticas gubernamentales }\end{array}$ \\
\hline $\begin{array}{l}\text { La propensión a } \\
\text { asumir riesgos }\end{array}$ & $\begin{array}{l}\text { El 64,3\% de los países que integran el } \\
\text { GEM poseen una tasa inferior al 10\% } \\
\text { de actividad emprendedora en etapas } \\
\text { tempranas del proceso de creación } \\
\text { (emprendedores nacientes y propietarios } \\
\text { gerentes de una actividad nueva hasta } \\
\text { tres años y medio) }\end{array}$ \\
\hline $\begin{array}{l}\text { La detección de } \\
\text { oportunidades de } \\
\text { negocio }\end{array}$ & $\begin{array}{l}\text { En la gran mayoría de países desarrollados, } \\
\text { la creación de empresas se debe } \\
\text { principalmente al descubrimiento de } \\
\text { oportunidades de negocio. En cambio, en } \\
\text { los países en vías de desarrollo es fruto de } \\
\text { la necesidad que experimentan algunos } \\
\text { individuos de cambiar su statu quo }\end{array}$ \\
\hline
\end{tabular}

Fuente: Informes del proyecto GEM referidos al periodo comprendido entre el año 2003 y el año 2006 (ambos incluidos).

En la sección siguiente se procederá a describir la investigación empírica llevada a cabo y los principales resultados obtenidos, lo que permitirá finalizar el presente trabajo con la discusión de los principales resultados y recomendaciones para la gestión.

\section{METODOLOGÍA Y ANÁLISIS DE RESULTADOS}

Con objeto de analizar la influencia de las motivaciones del emprendedor derivadas de la imagen social, la percepción del riesgo y la detección de oportunidades en la consolidación empresarial, se han planteado dos modelos de regresión logarítmicos. La estimación de dichos modelos se ha realizado con datos procedentes del proyecto GEM. Dichos datos han sido obtenidos en 14 países durante el periodo comprendido entre el año 2003 y el año 2006 (ambos inclusive) por medio de una encuesta telefónica realizada a emprendedores cuyas edades se sitúan entre los 18 y los 64 años (ver Cuadro 2).

Cuadro 2. Ficha técnica del cuestionario.

\begin{tabular}{|l|l|}
\hline Universo & $\begin{array}{l}\text { Emprendedores residentes en España, } \\
\text { Argentina, Estados Unidos, Suráfrica, Grecia, } \\
\text { Holanda, Bélgica, Francia, Hungría, Italia, } \\
\text { Reino Unido, Dinamarca, Noruega y Alemania } \\
\text { con edades comprendidas entre los 18 y los } \\
64 \text { años (ambos inclusive) }\end{array}$ \\
\hline $\begin{array}{l}\text { Selección } \\
\text { de la } \\
\text { muestra }\end{array}$ & $\begin{array}{l}\text { Procedimiento de muestreo probabilístico> } \\
\text { Muestreo polietápico: } \\
\text { (1) Selección aleatoria de ciudades y y } \\
\text { ámbito y cuotas de población residente; } \\
\text { (2) obtención aleatoria de los números de } \\
\text { teléfono correspondientes al municipio; } \\
\text { (3) selección de individuo }\end{array}$ \\
\hline Metodología & $\begin{array}{l}\text { Encuesta telefónica asistida por ordenador } \\
\text { (sistema CATI) }\end{array}$ \\
\hline $\begin{array}{l}\text { Nivel de } \\
\text { confianza }\end{array}$ & 95\% \\
\hline
\end{tabular}

Fuente: Informes del proyecto GEM referidos al periodo comprendido entre el año 2003 y el año 2006 (ambos incluidos).

En el primer modelo se ha introducido como variable endógena el porcentaje de población activa que es propietario-gerente de una actividad consolidada por año y por país (ESTBBUit), y como variables exógenas incluye, por año y por país, los porcentajes de respuesta afirmativos a las siguientes preguntas: (1)en su país, ¿la mayoría de las personas considera que poner en marcha un negocio es una buena opción de carrera profesional? (NBGOODit); (2) el miedo al fracaso, ¿le impediría empezar un nuevo negocio? (FRFAILit); (3) y en su país, ¿los medios de comunicación prestan mucha atención al fenómeno emprendedor? (NBMEDIit); (4) ¿en su país, se asocia un alto estatus a los empresarios que tienen éxito? (NBSTATit). Asimismo, el segundo modelo contiene la misma variable endógena y como variables exógenas incluye los índices de actividad emprendedora total -por motivos de 
necesidad ${ }^{4}$ - para mujeres (TEAFNEit) y hombres (TEAMNEit) - y de oportunidad ${ }^{5}$ - para mujeres $^{2}$ (TEAFOPit) y hombres (TEAMOPit)- por año y por país (véanse los Modelos 1 y 2).

$$
\begin{aligned}
& \log \left(\text { ESTBBU }_{i t}\right)=\alpha_{i}+\beta \log \left(\text { NBGOOD }_{i t}\right)+ \\
& \delta \log \left(\text { FRFAIL }_{i t}\right)+\gamma \log \left(\text { NBMEDI }_{i t}\right)+ \\
& \eta \log \left(\text { NBSTAT }_{i t}\right)+\varepsilon_{i t} \\
& \log \left(\text { ESTBBU }_{i t}\right)=\alpha_{i}+\beta \log \left(\text { TEAFN }_{i t-1}\right)+ \\
& \delta \log \left(\text { TEAFOP }_{i t-1}\right)+\gamma \log \left(\text { TEAMNE }_{i t-1}\right)+ \\
& \eta \log \left(\text { TEAMOP }_{i t-1}\right)+\varepsilon_{i t}
\end{aligned}
$$

De este modo, mientras que el modelo 1 trata de recoger la influencia de aspectos relacionados con la imagen social y la percepción del riesgo del empresario sobre el fenómeno de consolidación empresarial, el modelo 2 se centra en analizar el impacto de la creación de empresas por motivo oportunidad y por motivo necesidad en la consolidación empresarial.

Seguidamente, la Tabla 1 muestra los resultados de las estimaciones obtenidas respecto de la consolidación de las iniciativas emprendedoras durante el periodo 2003-2006 de los factores imagen social del empresario y percepción de riesgo.

Estos resultados ponen de manifiesto cómo las variables explicativas de imagen social y percepción del riesgo son capaces de explicar un 89,89\% de la variabilidad de la variable de consolidación empresarial considerada. Más concretamente, los parámetros asociados a las variables "emprender es una buena elección de carrera" y "el miedo al fracaso es un obstáculo para emprender" resultan positivos y estadísticamente significativos (elasticidades de $1,073 \%$ y $0,484 \%$ respectivamente). Estos hallazgos ponen de manifiesto cómo considerar la creación de una empresa como una buena opción profesional

4 El índice de actividad emprendedora total por motivo de necesidad incluye aquellos/as emprendedores/as nacientes (hasta tres meses de actividad) o propietarios/as-gerentes de una actividad nueva (hasta tres años y medio) en los que se observa la necesidad de cambiar el statu quo.

5 El índice de actividad emprendedora total por motivo de oportunidad incluye aquellos/as emprendedores/as nacientes (hasta tres meses de actividad) o propietarios/as-gerentes de una actividad nueva (hasta tres años y medio) que descubren una oportunidad de negocio.
Tabla 1. Estimación del primer modelo: consolidación de un negocio por imagen social y por percepción del riesgo.

Variable Dependiente: $\operatorname{LOG}\left(\operatorname{ESTBBU}_{t}\right)$

Método de estimación del Panel: Mínimos Cuadrados Generalizados (EGLS) con ponderaciones en las secciones

\begin{tabular}{|c|c|c|c|}
\hline Variable & Coef. & Est. $t$ & Valor $p$ \\
\hline $\mathrm{C}$ & 0,670662 & 0,540206 & 0,594 \\
\hline LOG(NBGOOD $\left.{ }_{t-1}\right)$ & 1,073678 & 3,160243 & 0,0042 \\
\hline${\text { LOG}\left(\text { FRFAIL }_{\mathrm{t}-1}\right)}$ & 0,484333 & 3,788831 & 0,0009 \\
\hline LOG(NBMEDI $\left.I_{t-1}\right)$ & $-0,049561$ & $-0,547582$ & 0,589 \\
\hline LOG$\left._{\left(N_{B S T A T}\right.}\right)_{\mathrm{t}-1}$ & $-1,212865$ & $-2,498288$ & 0,0197 \\
\hline \multicolumn{4}{|c|}{ Estadísticos no ponderados } \\
\hline R-cuadrado & 0,89899 & $\begin{array}{c}\text { Media } \\
\text { de la var. } \\
\text { dependiente } \\
\end{array}$ & 1,56135 \\
\hline $\begin{array}{l}\text { Suma de } \\
\text { cuadrados de los } \\
\text { residuos }\end{array}$ & 1,36472 & $\begin{array}{c}\text { Estadístico } \\
\text { de Durbin-- } \\
\text { Watson }\end{array}$ & 3,74956 \\
\hline
\end{tabular}
cruzadas

Período temporal (ajustado): 2004-2006

Observaciones temporales incluidas: 3 después del ajuste

Secciones de corte transversal incluidas: 14

Total de observaciones en el panel (balanceado): 42

y las mayores percepciones de riesgo incrementan las posibilidades de que se consolide el proyecto empresarial.

Sin embargo, la variable "se asocia un alto estatus a los emprendedores que tienen éxito" (NBSTATt-1), aunque significativa, tiene una elasticidad inelástica. Por tanto, aunque esta variable se ha tenido en cuenta en el proceso de consolidación, no contribuye a fomentarlo. Asimismo, la variable (NBMEDIt-1) "los medios de comunicación prestan mucha atención al fenómeno emprendedor", además de ser inelástica, es no significativa. Por tanto, estas dos variables no han desempeñado un efecto relevante en el proceso de consolidación empresarial.

A continuación se muestran los resultados de las estimaciones del modelo 2 (véase Tabla 2).

En este caso, las variables explicativas del modelo que recogen la creación de empresas por motivo de oportunidad y de necesidad explican un $91,54 \%$ en la varianza de la variable de consolidación empresarial que se ha tenido en cuenta. El análisis de los resultados recogidos en la Tabla 2 evidencia cómo la consolidación empresarial en 
Tabla 2. Resultados de la estimación del segundo modelo: consolidación de un negocio por motivos de necesidad y de oportunidad.

\begin{tabular}{|c|c|c|c|}
\hline $\mathrm{Va}$ & pendiente: I & $\mathrm{OG}(\mathrm{ES}$ & \\
\hline $\begin{array}{l}\text { Método de estimo } \\
\text { Generalizados (EG) }\end{array}$ & $\begin{array}{l}\text { ción del Pant } \\
\text { S) con ponde } \\
\text { cruzada }\end{array}$ & $\begin{array}{l}\text { l: Mínimos Cuac } \\
\text { raciones en las s }\end{array}$ & $\begin{array}{l}\text { rados } \\
\text { ecciones }\end{array}$ \\
\hline Período ter & tporal (ajust & do): 2004-2006 & \\
\hline Observaciones tem & orales inclui & das: 3 después d & lajuste \\
\hline Secciones de & corte transv & rsal incluidas: 1 & \\
\hline Total de obser & iones en el p & anel (balanceads & ): 42 \\
\hline Variable & Coef. & Est. T & Valor $p$ \\
\hline $\mathrm{C}$ & 0,899366 & 8,685019 & 0,0000 \\
\hline LOG(TEAFOPt-1) & $-0,187062$ & $-22,123780$ & 0,0000 \\
\hline LOG(TEAFNEt-1) & $-0,103884$ & $-3,974760$ & 0,0006 \\
\hline LOG(TEAMOPt-1) & 0,461107 & 7,503057 & 0,0000 \\
\hline LOG(TEAMNEt-1) & $-0,156273$ & $-5,712239$ & 0,0000 \\
\hline & no & derados & \\
\hline R-cuadrado & 0,91545 & $\begin{array}{c}\text { Media } \\
\text { de la var. } \\
\text { dependiente }\end{array}$ & 1,56135 \\
\hline $\begin{array}{l}\text { Suma de cuadrados } \\
\text { de los residuos }\end{array}$ & 1,14239 & $\begin{array}{l}\text { Estadístico de } \\
\text { Durbin-Watson }\end{array}$ & 3,47866 \\
\hline
\end{tabular}

los 14 países y periodo temporal considerados se basa principalmente en el descubrimiento y aprovechamiento de oportunidades de negocio $-\mathrm{y}$ no tanto en motivos de necesidad-, especialmente por parte de emprendedores masculinos (elasticidad de $0,461 \%$ ). No obstante, las iniciativas por necesidad de hombres y de mujeres, aunque significativas, resultan inelásticas $(-0,156 \%$ y $-0,103 \%$ respectivamente), al igual que las iniciativas femeninas por oportunidad (elasticidad de $-0,187 \%$ ).

\section{CONCLUSIONES Y RECOMENDACIONES PARA LA GESTIÓN}

La investigación realizada ha permitido identificar los factores motivacionales del emprendedor-referidos fundamentalmente a características del propio emprendedor y de su entorno- que determinan de manera significativa la consolidación empresarial en una muestra de los países que conforman el entorno GEM. En particular, los resultados obtenidos sugieren la existencia de una influencia significativa de diversas variables motivadoras tanto del empresario como del entorno en la probabilidad de consolidación empresarial.
Respecto a la imagen social del empresario, se ha corroborado que la variable "emprender es una buena elección de carrera" es la variable que mayor influencia ejerce sobre la consolidación empresarial. Sin embargo, no sucede lo mismo con la variable "los medios de comunicación prestan mucha atención al fenómeno emprendedor". Una posible explicación a este hecho puede encontrarse en el tipo de políticas implementadas en los países considerados del entorno GEM para incentivar la creación de empresas. En efecto, estos resultados parecen indicar que los medios de comunicación han podido no tenerse en cuenta por los agentes encargados de trazar las políticas de desarrollo local/regional. Por tanto, para incrementar la tasa de creación de empresas no bastaría únicamente con el diseño de programas de ayuda pública; para su aprovechamiento, resultaría necesario que la sociedad conociera la existencia y ventajas de los mismos.

Asimismo, los hallazgos obtenidos han puesto de manifiesto cómo la percepción del riesgo influye significativa y positivamente en la consolidación empresarial. Este resultado resulta consistente con aquellos trabajos previos que postulan un efecto positivo de la percepción del riesgo en la consolidación empresarial, que podría deberse al compromiso personal y empresarial más elevados que puede inducir en el emprendedor.

Asimismo, en relación a las motivaciones oportunidad frente a necesidad se ha detectado que existe una influencia positiva y significativa del motivo oportunidad en la consolidación empresarial, lo cual resulta coherente con algunos trabajos previos de la literatura.

Finalmente señalar que el modelo integrador planteado, que incorpora motivaciones del empresario y el entorno, posee un elevado poder explicativo de la consolidación empresarial. Lo cual pone de manifiesto la complementariedad existente entre los factores del empresario y el entorno en el impulso a la creación y posterior consolidación del proyecto empresarial.

De este estudio se han derivado importantes contribuciones al campo de la creación de empresas. Por un lado, frente a la excesiva fragmentación de la investigación en creación de empresas se 
ha planteado un modelo de carácter integrador. Además, se ha diferenciado el tipo de motivaciones de hombres y mujeres que mayor impacto ejercen en la consolidación empresarial. Por otro lado, se ha estudiado el fenómeno de la consolidación empresarial entre países, lo cual resulta especialmente novedoso teniendo en cuenta los trabajos previos que han analizado conjuntamente empresarios nacientes y consolidados de una misma cultura.

Cabe destacar que la principal limitación de este trabajo está relacionada con los constructos que se han incluido en el modelo causal. Y es que, a pesar de que pueden influir en la consolidación empresarial, constituyen tan sólo una parte de la gran diversidad de antecedentes y consecuencias que podrían tenerse en cuenta.

Asimismo, la revisión de la literatura realizada y los resultados obtenidos en este estudio sugieren la necesidad de seguir indagando en el estudio de los factores que determinan la consolidación empresarial. En este sentido, se deberían incorporar nuevas variables al modelo (e.g., la experiencia, los programas de ayuda pública, las expectativas de crecimiento del negocio y los valores culturales entre otros), a la vez que sería interesante profundizar en la investigación de sinergias entre las variables.

Por último, es posible señalar una serie de implicaciones tanto para los empresarios como para los agentes encargados de trazar políticas de desarrollo económico y creación de empresas. En relación a los primeros, los resultados muestran la conveniencia de crear empresas a partir de la detección de oportunidades en el entorno que posean potencial de innovación, logrando de este modo el crecimiento y la consolidación empresariales. En relación a los segundos, señalar que los resultados obtenidos pueden orientar a estos agentes sobre los factores del emprendedor y el entorno que deben potenciarse para incrementar la tasa de creación de empresas en un entorno geográfico específico como son: las normas sociales y culturales de apoyo a la creación y consolidación empresarial, y ciertas políticas de apoyo gubernamental encaminadas al aprovechamiento de los programas de ayuda pública a través de la difusión de los medios de comunicación.

\section{REFERENCIAS}

[1] GEM. Informe ejecutivo 2003 España. Instituto de Empresa. 2003

[2] GEM. Informe ejecutivo 2004 España. Instituto de Empresa. 2004.

[3] GEM. Informe ejecutivo 2005 España. Instituto de Empresa. 2005.

[4] GEM. Informe ejecutivo 2006 España. Instituto de Empresa. 2006.

[5] D. North and D. Smallbone. "Developing entrepreneurship and enterprise in Europe's peripheral rural areas: Some issues facing policy-makers". European Planning Studies. Vol. 14, Issue 1, pp. 41-60. 2006.

[6] P.H. Wilken. "Entrepreneurship. A comparative historical study". Alex Publishing Corporation. 1979.

[7] L.P. Dana. "Creating entrepreneurs in India". Journal of Small Business Management. Vol. 38, Issue 1, pp. 86-91. January, 2000.

[8] Y. Chen and D. Touve. "Conformity, political participation, and economic rewards: The case of Chinesse private entrepreneurs". Asian Pacific Journal of Management. On line November 6, 2009.

[9] G.N. Prabhu. "Social entrepreneurial leadership". Career Development International. Vol. 4, Issue 3, pp. 140-145. 1999.

[10] A. Shapero. "Some Social dimensions of entrepreneurship". In C.A. Kent, D.L. Sexton and K.H. Vesper (Eds.). Encyclopedia of entrepreneurship. Prentice Hall. Englewood Cliffs, New Jersey, USA. 1982.

[11] D.R. Gnyawali and D.S. Fogel. "Environments for entrepreneurship development: Key dimensions and research implications". Entrepreneurship: Theory and Practice. Vol. 18, Issue 4, pp. 43-62. 1994.

[12] L.W. Busenitz and C. Lau. "A cross-cultural cognitive model of new venture creation". Entrepreneurship: Theory and Practice. Vol.20, Issue 4, pp. 25-39. 1996.

[13] M. Low and I.C. McMillan. "Entrepreneurship: Past research and future challenger". Journal of Management. Vol. 14, Issue 2, pp. 139-161. 1988.

[14] L.E. Palich and D.R. Bagby. "Using cognitive theory to explain entrepreneurial risk-taking: Challenging conventional wisdom". Journal of Business Venturing. Vol. 10, Issue 6, pp. 425438. 1995. 
[15] J.A. Timmons. "The entrepreneurial mind". Brick House Publishing company. Acton, Massachusetts, USA. 1989.

[16] M.S. Wood and J.M. Pearson. "Taken on Faith? The Impact of Uncertainty, Knowledge Relatedness, and Richness of Information on Entrepreneurial Opportunity Exploitation". Journal of Leadership and Organizational Studies. Vol. 16, Issue 2, pp. 117-130. 2009.

[17] P.F. Drucker. "Innovation and entrepreneurship". Harper and Row. New York, N.Y., USA. 1985.

[18] D. Holtz-Eakin, D. Joulfaian and H.S. Rosen. "Entrepreneurial Decisions and Liquidity Constraints". RAND Journal of Economics. The RAND Corporation. Vol. 25, Issue 2, pp. 334-347. 1994.

[19] D.C. McClelland. "La sociedad ambiciosa. Factores psicológicos en el desarrollo económico". Guadarrama, Madrid. 1961.

[20] J. Arrazola. "Hacia un nuevo modelo: la empresa global integrada". Universia Business Review. Segundo trimestre No 014, pp. 108118. 2007.

[21] P. Bell. "The new entrepreneurship", Vital speeches of the day. Vol. 67, Issue 18, pp. 572575. 2001.

[22] I. Kirzner. "Creatividad, capitalismo y justicia distributiva". Unión Editorial S.A., Primera Editorial. Madrid, España. 1995.

[23] S. Venkataraman. "The distinctive domain of entrepreneurship research: an editor's perspective". In J. Katz and R. Brockhaus (Ed.). Advances in Entrepreneurship, Firm Emergence and Grown. JAI Press. Greenwich, CT, USA. 1997.

[24] J.A. Schumpeter. "Teoría del desenvolvimiento económico". Una investigación sobre ganancia, capital, crédito, interés y ciclo económico. Tercera Edición. Fondo de Cultura Económica. México. 1963.

[25] B.A. Kirchhoff. "Creative destruction among industrial firms, in the United States". Small Business Economics. Vol. 1, pp. 161-173. 1989.

[26] B.A. Kirchhoff. "Entrepreneurship's contribution to economics". Entrepreneurship Theory and Practice". Vol. 16, Issue 2, pp. 93-112. 1991.

[27] P.F. Drucker. "La disciplina de la innovación". En Creatividad e Innovación. Harvard Business Review, Ediciones Deusto S.A. Bilbao. España. 2000.

[28] A. Moraleda. "La innovación, clave para la competitividad empresarial". Universia Business Review, primer trimestre $\mathrm{N}^{\circ} 001$, pp. 128-136. 2004.

[29] T.M. Begley and D.P. Boyd. "Psychological characteristics associated with performance in entrepreneurial firm and smaller businesses". Journal of Business Venturing. Vol. 2, pp. 79-93. 1987.

[30] G.C. O'Connor and M.P. Rice-Hao Ho. "Opportunity recognition and breakthrough innovation in large established firms". California Management Review. Vol. 43, Issue 2, pp. 95-116. 2001.

[31] J. Craig and N. Lindsay. "Quantifying 'gut feeling' in the opportunity recognition process". Frontiers of Entrepreneurship Research. 2001. Date of visit: March 1, 2010. URL: http:// www.babson.edu/

[32] A. Cuervo. "Dinámica empresarial y consolidación sectorial". Universia Business Review. Primer trimestre $\mathrm{N}^{\circ} 001$, pp. 96105. 2004. URL: http://www.babson.edu/ 